\title{
Parameter estimation using Bayesian approach for a model of Pressure Peaking Phenomena-ignited $\mathrm{H}_{2}$ releases.
}

\author{
Agnieszka Weronika Lach Torbjørn Grande Østby \\ Process, Energy and Environmental Technology, University of South-Eastern Norway, Kjølnes Ring 533916 Norway, \\ Agnieszka.Lach@usn.no \\ Torbjorn.G. Ostbyausn.no
}

\begin{abstract}
The aim of this paper is to estimate the parameters of the model of Pressure Peaking Phenomena (PPP). This project focuses on the investigation of the overpressures arising from the ignited hydrogen releases in $14.9 \mathrm{~m}^{3}$ enclosure (explosive chamber) through a $4 \mathrm{~mm}$ nozzle. The various ventilation areas and mass flow rates were applied in 31 tests. The controlled variables for experiments are mass flow rate (MFR, $\mathrm{g} / \mathrm{s})$, ventilation area $\left(A_{v}, m^{2}\right)$ and time of hydrogen releases $(t, s)$. The Bayesian approach was implemented in the parameter estimation using Markov chain Monte Carlo method for simulations. The discharge coefficient and heat loss coefficient has been analyzed and gave by posterior distribution.
\end{abstract}

Keywords: pressure peaking phenomena, hydrogen safety, Bayesian analysis, parameter estimation, MCMC.

\section{Introduction}

Hydrogen releases in confined spaces are bringing new hazards into underground transportation systems. Unexpected hydrogen releases in confined spaces can result in dynamic overpressures with characteristic peaks. The phenomenon called pressure peak phenomena is distinct for hydrogen and occurs while introducing gas with lower density than the gas inside the enclosure (Brennan and Molkov, 2018). The characteristic transient pressure can be observed during releases with a high molar flow in combination with a relatively low ventilation area.

In a study by Makarov et al. (2018) a model for hydrogen jet fires from the TPRD (Thermal activated Pressure Relief Devices) was presented, which was demonstrated to be consistent with the experimental observations on in $\sim 1 \mathrm{~m}^{3}$ enclosure. The model predicted much higher overpressures compared to the unignited releases. The model consists of the volumetric mass balance of the gasses in the enclosure during a combustion process. They applied a perfect mix assumption and included adiabatic temperature in the calculations. The results of their simulation for garagelike enclosures showed the risk of enclosure collapse in a few seconds. The parameters used for the simulation were typical TPRD diameter and low ventilation area (commonly used in UK and France). Further numerical study of pressure peaking from ignited hydrogen releases was performed by Hussein et al. (2018). Their simulations were performed for small scale enclosures (experimental result from $1 \mathrm{~m}^{3}$ enclosure) and real scale (garage-like) for the common use of TPRD diameters. The model used ANSYS ICEM CFD (Computational Fluid Dynamics) to generate geometries for both studied cases. The RANS (Reynolds-Average Navier-Stokes) conservation equations were used for solving energy, mass, species and momentum, turbulent model, radiation, and combustion model. The EDC (Eddy Dissipation Concept) model was used to solve the combustion process and DO (Discrete Oridantes) model to include the radiation process. Results demonstrated the relation between vent area and release rate. The big impact of heat transfer mechanisms in the prediction of the pressure peaking phenomenon was acknowledged. It was concluded that the current TPRD diameters may result in a significantly dangerous situation in underventilated enclosures. The heat transfer mechanisms (radiation and conduction) has been investigated (Hussein et al., 2018). The assumption of adiabatic walls showed high over predictions compared to simulations where both radiation and conduction were included. The heat transfer has been computationally demonstrated but due to the small scale of experiments $(2 \mathrm{~s}$ experimental run) couldn't be physically observed. The heat transfer in the system is found to be important and needs further investigation.

In this study, the model of ignited hydrogen releases developed at USN (University of South-Easter Norway) is presented for the first time. It is based on the problem approach presented by Brennan et al. (2010). The model was validated against large scale experiments in the explosion chamber, allowing to observe high overpressures (over $45 \mathrm{kPa}$ ). In this study, we use the Bayesian parameter estimation approach to estimate the model parameters, the discharge coefficient and the heat loss. The Bayesian method combines the information on a discrepancy between the model and the measurements given a set of parameter values (described by the likelihood) and the information available in the literature (described by the prior) through use of the Bayes rule to determine the probable values of the unknown parameters. An important distinction between 
the Bayesian method and other classical methods is that it can explicitly and consistently incorporates all the existing knowledge about the unknown parameters. The results of each parameter consistent with the measurements are represented by a posterior distribution. We use a Markov Chain Monte Carlo (MCMC) method to generate samples from the posterior, which can be used for estimation of the unknown parameters. In recent years, the popularity of the MCMC methods has been on the rise (Vrugt, 2016). The prior distribution is chosen based on information found in the literature in order to impose bounds on the parameter space. The prior and posterior densities have been graphically presented as a normal distribution function as in studies on combustion kinetics models (Wang et al., 2020).

The main goal is to create a probability density function (pdf) for parameters giving the most accurate model.

Using Bayesian analysis allows us to understand the parameters' influence on pressure dynamics in the system hence improve the model. The pressure peaking model is designated to be an engineering tool for safety engineers. The accurate parameter estimation plays a significant role in its development.

The investigation of occurring overpressures from ignited hydrogen releases in confined spaces is part of pre-normative research for the safety of hydrogen fuel vehicles and transport through tunnels and similar confined spaces (Hy-Tunnel CS).

\section{Case set up and methodology}

\subsection{Experimental setup and materials}

All experiments were performed in a steel explosion with a total volume of $14.9 \mathrm{~m}^{3}$. The explosion chamber's walls in total have five vents of $80 \mathrm{~mm}$ diameter each $\left(0.005027 \mathrm{~m}^{2}\right)$. The vent in the middle of the front wall was used for the hydrogen and propane pipes. Three of the vents were used to vary the passive ventilation area. The flanges were used to fully close/open the vents with gasket ensuring no leakage. The specifics of the releases were obtained with hydrogen mass flow through a stainless pipe outlet located in the center of the chamber's floor to vertically discharge hydrogen jets fires.

The Coriolis mass flow meter and the pneumatic valve were mounted to measure and control hydrogen releases. Oscilloscope Sigma has recorded pressure and mass flow rate from Coriolis mass flow. The complete overpressure development was measured with Kulite pressure transducer XTM - 190-50A. Oscilloscope Gen3i recorded the overpressure constantly with parallel measurement initiated by the voltage signal (with $25 \mathrm{ks} / \mathrm{s}$ ). In the table below are listed the uncertainties of measurement.

Table 2. Standard deviations of instruments

\begin{tabular}{|l|c|}
\hline Pressure sensor & $\begin{array}{c} \pm \% \text { FSO BFSL } \\
250 \text { point }(0.025 \mathrm{sec}) \text { filter }\end{array}$ \\
\hline Mass flow sensor & $\pm 0.5 \%$ of a flow rate \\
\hline Thermocouples type K & $\pm 0.75 \%$ \\
\hline
\end{tabular}

\subsubsection{Data set}

The experiments were designed for three different ventilation areas and with a variety of mass flow rates The purpose of those experiments was to validate the model of PPP. To observe overpressure the mass flow rate of discharged hydrogen into an enclosure has to be relatively high while the ventilation area has to be relatively small (Makarov et al., 2018). The chosen combination of vent area and mass flow rates is based on previous unignited experiments (Lach, 2019) and studies on PPP (Hussein et al., 2018). The pressure peak phenomenon for hydrogen jet fires in the $14.9 \mathrm{~m}^{3}$ enclosure was successfully observed during all 31 experiments. Experiment 11 described in Table 1 will be used to present methods of the Bayesian approach for parameter estimation.

The measured pressure of experiment 11 is presented in Figure 1. The uncertainty of the Kulite sensor together with the sampling time, result in uncertainty $-/+0.22$ $\mathrm{kPa}$.

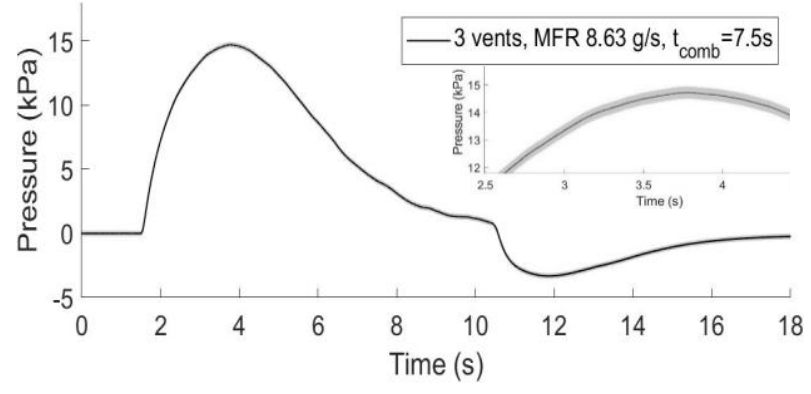

Figure 1. Exp 11; Overpressure for MFR $8.63 \mathrm{~g} / \mathrm{s}$ with 3 open vent with total vent area $0.0164 \mathrm{~m}^{2}$. Measurement uncertainty $0.22 \mathrm{kPa}$.

Table 1 Experimental results: $\mathrm{H}_{2}$ releases through $4 \mathrm{~mm}$ nozzle in $14.9 \mathrm{~m}^{3}$ enclosure with ventilation area: 1 open vent $=0.0055 \mathrm{~m}^{2}, 2$ vent open $=0,0109 \mathrm{~m}^{2}, 3$ vents open $=0.0164 \mathrm{~m}^{2}$.

\begin{tabular}{|c|c|c|c|c|c|}
\hline \multicolumn{4}{|c|}{ Setup } & \multicolumn{2}{|l|}{ Measured } \\
\hline $\begin{array}{l}\text { Exp } \\
\text { nr }\end{array}$ & $\begin{array}{l}\mathrm{T}_{0} \text { in enclosure } \mathrm{H}_{2} \text { release } \\
{[\mathrm{K}]}\end{array}$ & $\begin{array}{l}\text { Open } \\
\text { vent }\end{array}$ & $\begin{array}{l}\text { Mass flow rate } \\
{[\mathrm{g} / \mathrm{s}]}\end{array}$ & $\begin{array}{l}\text { Experimental overpressure } \\
(\max )[\mathrm{kPa}]\end{array}$ & $\begin{array}{l}\text { Experimental underpressure } \\
(\max )[\mathrm{kPa}]\end{array}$ \\
\hline 11 & 7.50 & 3 & 8.63 & 14.70 & -3.30 \\
\hline
\end{tabular}




\subsection{Model of Pressure Peaking Phenomena- governing equation}

The model of pressure dynamics in the enclosure during ignited hydrogen releases (jet fires) is presented. In the calculations, the stoichiometric hydrogen combustion was applied where one mole of hydrogen requires 0.5 moles of oxygen: $\mathrm{H}_{2}+0.5\left(\mathrm{O}_{2}+3.76 \mathrm{~N}_{2}\right) \leftrightarrow \mathrm{H}_{2} \mathrm{O}+1.88 \mathrm{~N}_{2}$. The system of conservation equations described in this chapter provides a solution for the PPP model explaining mass balance and temperature based on the internal energy in the enclosure.

The mass balance for each species in the enclosure is described with the system of equations (1)-(6). The number of moles in the enclosure $n_{\text {tot }}$ is a sum of the number of moles of each species in the enclosure $n_{t o t}=\Sigma n_{i}$ :

$$
\begin{aligned}
& \frac{d n_{\mathrm{H}_{2}}}{d t}=\dot{n}_{i n \mathrm{H}_{2}}-\dot{n}_{o u t \mathrm{H}_{2}}+\dot{n}_{r x \mathrm{H}_{2}} \\
& \frac{d n_{\mathrm{O}_{2}}}{d t}=\dot{n}_{\text {inO }_{2}}-\dot{n}_{\text {outo }}+\dot{n}_{r x O_{2}} \\
& \frac{d n_{\mathrm{N}_{2}}}{d t}=\dot{n}_{\text {inN }_{2}}-\dot{n}_{\text {out } \mathrm{N}_{2}}+\dot{n}_{r x N_{2}} \\
& \frac{d n_{\mathrm{H}_{2} \mathrm{O}}}{d t}=\dot{n}_{\text {in }_{2} \mathrm{O}}-\dot{n}_{\text {out } \mathrm{H}_{2} \mathrm{O}}+\dot{n}_{r x \mathrm{H}_{2} \mathrm{O}}
\end{aligned}
$$

Where $\dot{n}_{i n, H_{2}}=\dot{m}_{i n} / M_{H_{2}}$ (hydrogen mass flow $\dot{m}_{i n}$ was measured with coriolis mass flow meter), given the initial conditions (at $\mathrm{t}=0$ ) $n_{\mathrm{H}_{2} .0}=0 . n_{O_{2} .0}=0.21$. $n_{\text {tot } .0} \cdot n_{N_{2} .0}=0.79 \cdot n_{\text {tot. } 0} \cdot n_{H_{2} O .0}=0$ and $n_{\text {tot. } .0}=$ $p_{0} \cdot \frac{V}{R T_{0}}$. If the pressure inside the enclosure will be lower than ambient pressure $\Delta \mathrm{p}<0.001$ that means the hydrogen mass flow into the enclosure was closed and $\dot{n}_{i n, H_{2}}=0$. Then the air from outside the chamber starts to flow into the enclosure through the vent area, means the $\dot{n}_{\text {out }, i}=0$ and:

$$
n_{\text {in }}=\frac{C_{\text {in }} \cdot A}{M_{\text {air }}} \sqrt{2 \cdot \Delta p \cdot \rho_{\text {air }}}
$$

Where $M_{\text {air }}$ is the molecular mass of air and $\rho_{\text {air }}$ is the density of air. The $C_{i n}$ and $A$ is the discharge coefficient and the vent area, respectively. Using equation (5) the $\dot{n}_{i n, i}$ can be calculated: $\dot{n}_{i n, O_{2}}=0.21$. $\dot{n}_{i n}$ and $\dot{n}_{i n, N_{2}}=0.79 \cdot \dot{n}_{i n}$ given the assumption that $\dot{n}_{i n, \mathrm{H}_{2} \mathrm{O}}=0$.

By balancing the combustion reaction equation, to burn all hydrogen $\dot{n}_{r x, H_{2}}=-\dot{n}_{i n, H_{2}}$ the oxygen needed will be: $\dot{n}_{r x, O_{2}}=0.5 \cdot \dot{n}_{r x, H_{2}}$. The produced water vapor then will be $\dot{n}_{r x, H_{2} O}=-\dot{n}_{i n, H_{2}}$, and $\dot{n}_{r x, N_{2}}=0$.

Equation (6) based on the steady-state in a compressible energy equation (Bernoulli equation) gives the molar flow out $n_{\text {out,tot }}$, which contains all the species in the enclosure from the initial stage and combustion products (no condensation of the water on the walls inside the chamber):

$$
n_{\text {out }, \text { tot }}=C \cdot A \sqrt{\frac{2 \cdot \Delta p \cdot n_{\text {tot }}}{V \cdot M_{\text {en }}}}
$$

Where $n_{\text {tot }}=\sum_{i} n_{i}$ is the number of moles in the enclosure, the molecular mass in the enclosure is then $M_{\text {en }}=\sum_{i} X_{i} M_{i}$ where $X_{i}=n_{i} / n_{\text {tot }}$ and $\dot{n}_{\text {out }, i}=X_{i}$. $n_{\text {out tot }}$ can be calculated. The $\mathrm{C}$ is the discharge coefficient and $\mathrm{A}$ is the ventilation area.

To calculate the pressure in the enclosure (12) the temperature has to be solved first. The internal energy $U$ for the system is equal to:

$$
\begin{aligned}
& \frac{d U}{d t}=\dot{H}_{\text {in }}-\dot{H}_{\text {out }}+\dot{Q}_{r x}-\dot{Q}_{\text {loss }} \\
& \text { And } \\
& \frac{d U}{d t}=\frac{d n \widehat{U}}{d t}
\end{aligned}
$$

With the assumption of specific internal energy $\widehat{U}$ :

$$
\widehat{U}_{i}=\widehat{U}_{r e f, i}+\int_{T_{r e f}}^{T} c_{v, i} d t
$$

Where $\widehat{U}_{\text {ref }, i}=0$ at $T_{\text {ref }}=298.15 \mathrm{~K}$ and $c_{v, i}$ is the molar heat capacity in a constant volume. Changing the internal energy to the temperature will result in a governing equation of temperature in the enclosure $T_{e n}$ :

$$
\begin{aligned}
& \quad \frac{d T_{e n}}{d t}=\frac{1}{\sum_{i} n_{i} c_{v, i}}\left(\dot{H}_{\text {in }}-\dot{H}_{\text {out }}+\dot{Q}_{r x}-\dot{Q}_{\text {loss }}-(T-\right. \\
& \left.\left.T_{r e f}\right) \cdot \sum_{i} c_{v, i} \frac{d n_{i}}{d t}\right)
\end{aligned}
$$

The $\dot{H}_{i n}=\sum_{i} \dot{n}_{i n . i} \widehat{H}_{i}$ is the sum of enthalpies of each species at $T=T_{i n}$. Using the same analogy the enthalpy of the system at $T=T_{e n}$ is the sum of enthalpies: $\dot{H}_{\text {out }}=\sum_{i} n_{\text {out } . i} \cdot \widehat{H}_{i}$.

The $c_{v, i}$ was calculated by reducing the heat capacity at constant pressure by the universal gas constant $c_{v, i}=$ $c_{p, i}-R$. The $c_{p, i} \dot{H}_{i n, i}$ and $\dot{H}_{o u t, i}$ where calculated with NASA polynomials (Mcbride et al., 1993) which includes thermodynamic data coefficients and enthalpy of formation $\widehat{H}_{f, i}$.

The $\dot{Q}_{r x}$ is the heat of formation. Since the water vapor is the only product $\dot{Q}_{r x}=\dot{Q}_{r x, \mathrm{H}_{2} \mathrm{O}}=\dot{n}_{r x \mathrm{H}_{2} \mathrm{O}}$. $\left(-\Delta \widehat{H}_{f_{H_{2} O}}\right)$ where the $\widehat{H}_{f_{H_{2} O}}$ is the enthalpy of formation of water vapor.

The $\dot{Q}_{\text {loss }}$ is the heat loss calculated with the major assumption of a simple heat transfer with no condensation of water: $\dot{Q}_{\text {loss }}=h_{\text {loss }} \cdot A_{\text {wall }} \cdot\left(T_{\text {en }}-\right.$ $\left.T_{\text {wall }}\right)$ where $A_{\text {wall }}$ is the surface area inside the enclosure and $h_{\text {loss }}$ is the heat transfer coefficient. When the pressure inside the enclosure will be lower than ambient pressure $\Delta p<0.001$ the cold air is entering into enclosure and heat transfer coefficient is assumed to be $h_{\text {loss }, 2}=0.5 * h_{\text {loss }}$.

The change of the number of moles in enclosure causes temperature change, expressed in equation (10) with $\left(\left(T-T_{r e f}\right) \cdot \sum_{i} c_{v, i} \frac{d n_{i}}{d t}\right)$. The temperature of the wall $T_{\text {wall }}$, was calculated with the major assumption that the whole wall is one thermal mass with the same temperature inside (i.e. no temperature gradient in the wall):

$$
\frac{d T_{\text {wall }}}{d t}=\frac{\dot{Q}_{\text {loss }}}{m_{\text {wall }} \cdot C_{\text {steel }}}
$$


Where $m_{\text {wall }}$ is the mass of walls and $C_{\text {steel }}$ is the heat capacity of the wall.

Solving the PPP model with conservation equations (1), (2), (3), (4), (10), (11) then allows to calculating pressure inside the enclosure with the ideal gas law (12):

$$
p_{e n}=\frac{n_{t o t} \cdot R \cdot T_{e n}}{V}
$$

\subsection{Bayesian framework}

A Bayesian approach with Monte Carlo simulation was used in estimating the parameters: the discharge coefficient, $C$, and the heat transfer coefficient, $h_{\text {loss }}$. The purpose of using Bayesian inference is to obtain new recognition about the parameters $\theta$. Whereas standard optimization techniques would estimate the optimal value for these parameters, Bayesian simulation instead estimates a distribution of probable values (based on including observed data and prior knowledge). Using a Bayesian approach makes sense given the uncertainties inherent in experiments and measurements.

The relationship between $\theta=\left[C, h_{\text {loss }}\right]$ and the measurement data $d$ is given according to Bayes' theorem, equation (13). $C$ and $h_{\text {loss }}$ are assumed to be independent.

$$
\begin{gathered}
p_{\text {post }}(\theta \mid d, M, I)=\frac{\mathcal{L}(d \mid \theta, M, I) p_{\text {prior }}(\theta \mid M, I)}{\int \mathcal{L}(d \mid \theta, M, I) p_{\text {prior }}(\theta \mid M, I) d \theta} \\
\propto \mathcal{L}(d \mid \theta, M, I) p_{\text {prior }}(\theta \mid M, I)
\end{gathered}
$$

Here $p_{\text {post }}(\theta \mid d, M, I)$ is the posterior probability for parameters $\theta$ given the measurement data $d, M$ representing the previously described model, and $I$ represents other unspecified information given in this paper, such as the experimental setup. The $p_{\text {post }}(\theta \mid d, M, I)$ expresses the updated knowledge of the $\theta$ given the $d, M, I$. Further, $p_{\text {prior }}(\theta \mid M, I)$ represents the prior probability distribution for parameters $\theta$ given the model $M$ and the information $I$ the initial belief about the $\theta$. Preliminary testing suggested that the value of $h_{\text {loss }}$ was between 20 and 40 , while the value of $C$ was between 0.6 and 1 . The prior distributions used are further detailed in the next subchapter. Finally, $\mathcal{L}(d \mid \theta, M, I)$ represents the likelihood (probability) of seeing the measurement data $d$ given the parameters $\theta$, the model $M$, and other information $I$. The Bayesian calculation will confront the simulation results of the earlier described model as a function of $\theta$ and $d, M, I$ to gather the pdf (probability density function) of the $\theta$ for the observed data.

Assuming the measurement uncertainties are normally distributed, the likelihood is given by the Gaussian function (Daly et al., 1995). As experiments consist of multiple measurements, this gives us equation (14), where $d_{i}$ (experimental overpressure) and $m_{i}$ (simulated overpressures) represent measurement data and model data at index $i$ (step), respectively. The sigma, $\sigma$ is the standard deviation of the measurement uncertainty (constant $\sigma$, Table 2).

$$
\mathcal{L}(d \mid \theta, M, I)=\prod_{i=1}^{n} \frac{1}{\sqrt{2 \pi \sigma^{2}}} \exp \left(-\frac{\left(d_{i}-m_{i}\right)^{2}}{2 \sigma^{2}}\right)
$$

Having the priors and likelihood allows the posterior pdf to be estimated through Monte Carlo simulation. This was done using the standard Metropolis Markov Chain Monte Carlo algorithm, which is further detailed in algorithm 1.

\section{Algorithm 1}

The Metropolis algorithm (adapted from (Kruschke, 2015)

1. Given: data $d$; prior distribution $p_{\text {prior }}(\theta \mid M, I)$;

likelihood function $\mathcal{L}(d \mid \theta, M, I)$; step standard deviation $\sigma ;$ number of steps $T$

2. Initialize $\theta^{0}$

3. For $i=1$ to $T$ :

4. Sample $\theta^{\prime} \sim \mathcal{N}\left(\theta^{i-1}, \sigma_{2}\right)$

5. Sample $u \sim \mathcal{U}(0,1)$

6. $\quad p_{\text {move }}=\min \left(1, \frac{\mathcal{L}\left(d \mid \theta^{\prime}, M, I\right) p_{\text {prior }}\left(\theta^{\prime} \mid M, I\right)}{\mathcal{L}\left(d \mid \theta^{i-1}, M, I\right) p_{\text {prior }}\left(\theta^{i-1} \mid M, I\right)}\right)$

7. If $p_{\text {move }} \geq u: \theta^{i}=\theta^{\prime}$; else: $\theta^{i}=\theta^{i-1}$

This is a sampling algorithm where samples producing a higher probability than the current sample is always accepted, while samples producing a lower probability than the current sample is sometimes accepted, depending on a randomly sampled value. This process is then repeated for a limited number of steps.

However, with high numbers of measurements, the likelihood has a tendency to vanish, as many values between 0 and 1 are multiplied. Using the natural logarithm is a natural way of overcoming this. Using the natural logarithm changes steps 6 and 7 of algorithm 1 in the following way. The fraction in step 6 is changed as shown in equation (15). Here $\ell$ refers to the loglikelihood, and $p_{\text {prior }}$ refers to the logarithm of the prior. Due to a high number of steps (100000) needed to describe our physical event the Gaussian distributions were assumed for the loglikelihood and the logprior. Then $\ell$ and $p_{\text {prior }}$ can be calculated as shown in equation (16) and (17). In equation (16), as in equation (14), $d_{i}$ and $m_{i}$ represent measurement data and model data at index $i$, respectively, and $\sigma$ is the standard deviation of the measurement uncertainty. In equation (17) $\theta_{j}$ represents the $j$-th parameter in $\theta$, and $\mu_{j}$ and $\sigma_{j}$ represent the expected value and standard deviation, respectively, for the prior for this parameter. Equation (17) is only valid if the parameters of $\theta$ are independent. The final change that needs to be made is to alter step 7 to compare $p_{\text {move }}$ to the value of $\ln (u)$ instead of $u$.

$$
\begin{aligned}
& \ln \left(\frac{\mathcal{L}\left(d \mid \theta^{\prime}, M, I\right) p_{\text {prior }}\left(\theta^{\prime} \mid M, I\right)}{\mathcal{L}\left(d \mid \theta^{i-1}, M, I\right) p_{\text {prior }}\left(\theta^{i-1} \mid M, I\right)}\right)= \\
& \ell\left(d \mid \theta^{\prime}, M, I\right)+p_{\text {prior }}\left(\theta^{\prime} \mid M, I\right) \\
& -\ell\left(d \mid \theta^{i-1}, M, I\right)-p_{\text {prior }}\left(\theta^{i-1} \mid M, I\right)
\end{aligned}
$$




$$
\begin{aligned}
& \ell(d \mid \theta, M, I)=k+\sum_{i=1}^{n}-\frac{\left(d_{i}-m_{i}\right)^{2}}{2 \sigma^{2}} \\
& p_{\text {prior }}\left(\theta^{i-1} \mid M, I\right)=l+\sum_{j=1}^{m}-\frac{\left(\theta_{j}-\mu_{j}\right)^{2}}{2 \sigma_{j}^{2}}
\end{aligned}
$$

\subsubsection{MCMC Setup}

There are parameters that must be set for the MCMC algorithm, including the number of steps $T$, the standard deviation of the step $\sigma_{2}$. Additionally, the prior distributions for $\theta$ must be specified. Based on this the MCMC was run with the parameters and priors shown in Table 3. The prior probability distributions for $C$ and $h_{\text {loss }}$ are also shown in Figure 2.

Table 3. MCMC parameters and prior distributions

\begin{tabular}{|c|c|}
\hline$T$ & 100000 \\
\hline$\sigma_{C}$ & 0.01 \\
\hline$\sigma_{h_{\text {loss }}}$ & 0.1 \\
\hline$p_{\text {prior }}(\theta \mid M, I)$ & $p_{\text {prior }}(C \mid M, I) \cdot p_{\text {prior }}\left(h_{\text {loss }} \mid M, I\right)$ \\
\hline$p_{\text {prior }}(C \mid M, I)$ & $\mathcal{N}(0.8,0.05)$ \\
\hline$p_{\text {prior }}\left(h_{\text {loss }} \mid M, I\right)$ & $\mathcal{N}(30,5)$ \\
\hline
\end{tabular}
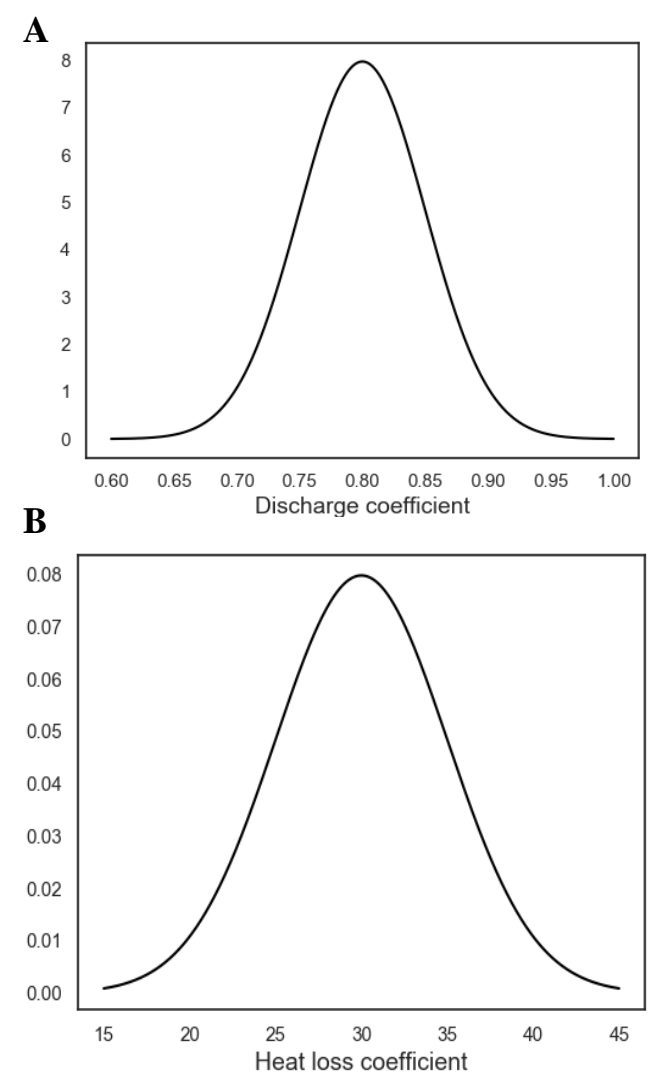

Figure 2. The prior probability distributions for A: discharge coefficient $\boldsymbol{C}$ and B: heat loss coefficient $h_{\text {loss }}$.

Additionally, some lead/lag compensation was implemented due to a time offset between the experimental and simulated data of approximately 4000 time-steps, consistent between experiments. It resulted in a significant difference during the rise and fall of the pressure response in the experiments.

The model was solved using solve nonstiff differential equations- medium order method ode45 (MathWorks, 2020), with a maximum time step of $0.5 \mathrm{~s}$.

\subsubsection{Evaluation of MCMC representativeness}

The representativeness and performance of the MCMC chains that were run were evaluated as described by Kruschke (2015). In this subchapter "the chains" refers to four chains run on experiment 11 .

A visual inspection of the chains' trajectory shows that after a burn-in period, all the chains had converged to the same area. The first 3000 steps of each chain were then removed, considered to be part of burn-in. With these steps removed the chains were seen to overlap and mix well, and distinguishing the different chains from one another was basically impossible. This indicates that none of the chains are stuck with all the chains sampling from the same region. The convergence of chains with the starting position given by the $\mathrm{X}$ marker is presented in Figure 3.

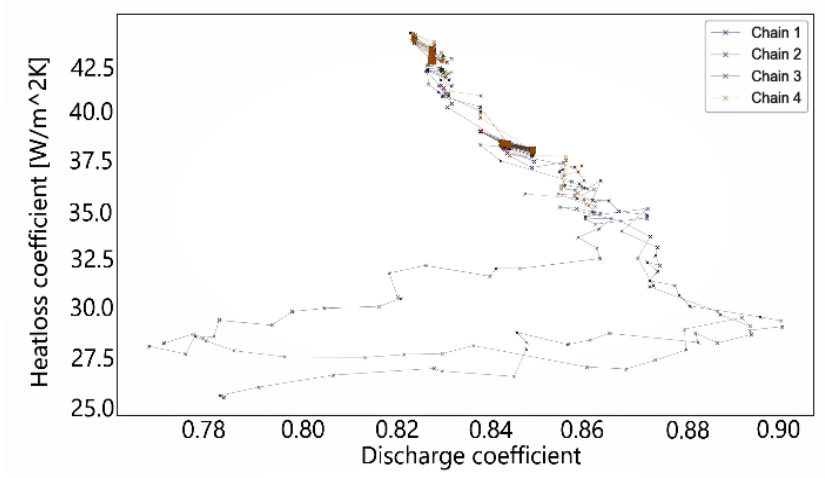

Figure 3. Convergence of 4 chains run in MCMC; exp 11.

The plots of marginal distributions were then created for the sampled parameter values for each chain, shown in Figure 4 and Figure 5. These density plots show high overlap, though with some difference in the peaks. Some difference in the density plot is to be expected, due to the finite number of samples drawn. The overlap between chains suggests the chains sample representative values from the posterior distribution. 


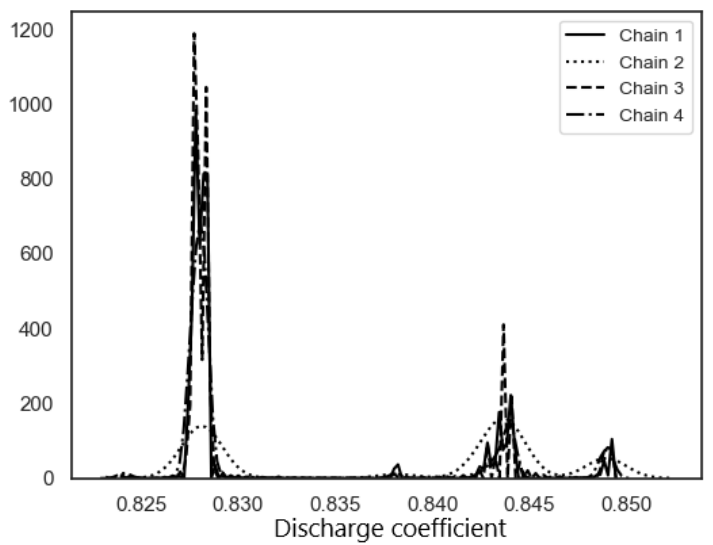

Figure 4. Density plot for parameter $\boldsymbol{C}$ for chain 1 through 4 for experiment 11 .

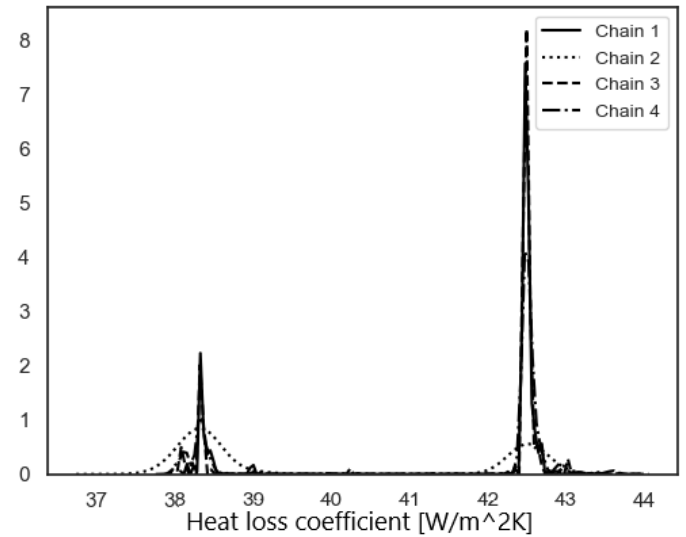

Figure 5. Density plot for parameter $\boldsymbol{h}_{\text {loss }}$ for chain 1 through 4 for experiment 11.

This evaluation of the MCMC gives us confidence in the results produced by the simulation. The evaluation shows no indication of poor representativeness nor low accuracy.

\subsubsection{Posterior predictive check}

If our model is adequate, then replication of the current experiment should generate data that are similar to the predictions made by the model. Let $y_{t}=y(t ; \theta)$ denote the predicted pressure given $\theta=(C, h)$. We are interested in $p\left(y_{t} \mid D, m, I\right)$, where $\mathrm{D}$ and $\mathrm{m}$ denote the experimental data from the previous run and the model output (section 2.2), respectively. It follows from the marginalization and the product rules that

$$
\begin{gathered}
p\left(y_{t} \mid D, m, I\right)=\int_{\Omega} p\left(y_{t}, \theta \mid D, m, I\right)= \\
\int_{\Omega} p\left(y_{t} \mid D, \theta, m, I\right) p(\theta \mid D, m, I) d \theta
\end{gathered}
$$

In case we know $m$ and $\theta$, it is reasonable to assume that previously acquired data and the prediction $y_{t}$ are conditionally independent. This means that

$$
p\left(y_{t} \mid D, \theta, m, I\right)=p\left(y_{t} \mid \theta, m, I\right)
$$

Thus, the posterior predictive density is given by

$$
p\left(y_{t} \mid D, m, I\right)=\int_{\Omega} p\left(y_{t} \mid \theta, m, I\right) p(\theta \mid D, m, I) d \theta
$$

The second term of the integrand, $p(\theta \mid D, m, I)$ is the posterior density, which has been previously determined. The first term of the integrand, $p\left(y_{t} \mid \theta, m, I\right)$, is the model density which depends on the model and the measurement noise. The model density has the same functional form as the likelihood for a single data point. Note that, in the case of model density, this function is a probability density with respect to $y_{t}$, with the parameter $\theta$ assumed to be known.

The expression for the posterior predictive density (20) shows that the uncertainty in the predicted pressure is due to two processes. The first one is contributed by the posterior density, which expresses the uncertainty about the true value of $\theta$. The second contribution is due to the measurement noise. Although, more experimental evidence can reduce the uncertainty about $\theta$, nevertheless, the measurement noise cannot be eliminated. In the maximum likelihood approach, it is assumed that the inferred value $\theta_{M L}$ for the unknown parameter is the true value and therefore the only source of uncertainty is the measurement noise. Indeed, in the context of eq. (20), in the maximum likelihood approach one claims that

$$
p(\theta \mid D, m, I)=\delta\left(\theta-\theta_{M L}\right)
$$

and hence, the integral in (20) reduces to

$$
p\left(y_{t} \mid D, m, I\right)=p\left(y_{t} \mid \theta_{M L}, m, I\right)
$$

As the evidence grows, we will become more confident about the true value of $\theta$ and hence the posterior density becomes narrower and in the limit it converges to (21). Nevertheless, as long as the uncertainty about the true value of $\theta$ is large, the Bayesian and maximum likelihood approaches will differ.

Due to difficulties to find an analytical expression for $y_{t}$, in general, a Monte Carlo based approach is more viable. The algorithm below describes the steps.

\begin{tabular}{l} 
Algorithm 1 \\
Generating $y_{t} ;$ for $i=1, \ldots, N$ repeat the following \\
steps \\
\hline $1 . \quad \theta_{i} \sim p(\theta \mid D, m, I)$ \\
$2 . \quad y_{i} \sim p\left(y \mid \theta_{i}, m, I\right)$
\end{tabular}

Applying this algorithm for large $\mathrm{N}$ (equal to the number of MCMC samples), the empirical distribution of the samples $y_{i}$ will approximate $p\left(y_{t} \mid D, m, I\right)$.

\section{Results and discussion}

Hydrogen jet fires are causing high overpressure due to the high amount of released energy in a very short time. The molar balance and temperature in the enclosure described in the methodology explained the pressure dynamic.

In the model, the mass balance is crucial for the accuracy of overpressure prediction. Therefore the discharge coefficient used in the calculation of molar flow through the vents (in and out) needs to be 
investigated. A discharge coefficient is a dimensional number representing flow and pressure loss through the orifice. Is a function of Reynold number $C=f(R e)$ while the $R e$ is a function of the flow rate, hence the $E_{\text {loss }} \sim u^{2}$. Some assumptions have been used for different scenarios but when the discharge coefficient is unknown or uncertain it has to be found experimentally. For the computing methods, it is recommended to assume $\mathrm{C}=1$ (Crowl and Louvar, 2011). From the previous study on PPP it is known that $\mathrm{C}=1$ is an assumption that can't be applied, and lower values showed better accuracy. The reasoning of choosing the values of $\mathrm{C}$ in the work of Hussein et al., (2018) and Makarov et al., (2018) was based on the literature knowledge and validated against their experiments. The values presented in their work are not. It can be due to different experimental setups and mass flow rates. In the mentioned studies the heat transfer shows its importance for the pressure dynamics. Nevertheless, the heat loss has been neglected in the model (due to the small scale of experiments).

In the model presented in this paper a simple solution of the heat loss is used. The water condensation hasn't been included in the numerical simulations. The heat loss described in section 2.2 determines the rate of heat transfer through the walls.

The MCMC evaluation results in the posterior distributions. Experiment 11 has been chosen to presents the parameter estimation analysis. In the figure below (Figure 6) the posterior pdf is presented. The clusters you can see on the plots represent the area of 'the best' $\theta$. Experiment 11 resulted in bimodal posterior distributions. By looking into the density plots of $\mathrm{C}$ and h_loss (Figure 4 and Figure 5) the two major peaks are clearly visible. Two clusters represent two areas of $\theta$ value which are consistent with the experimental results.

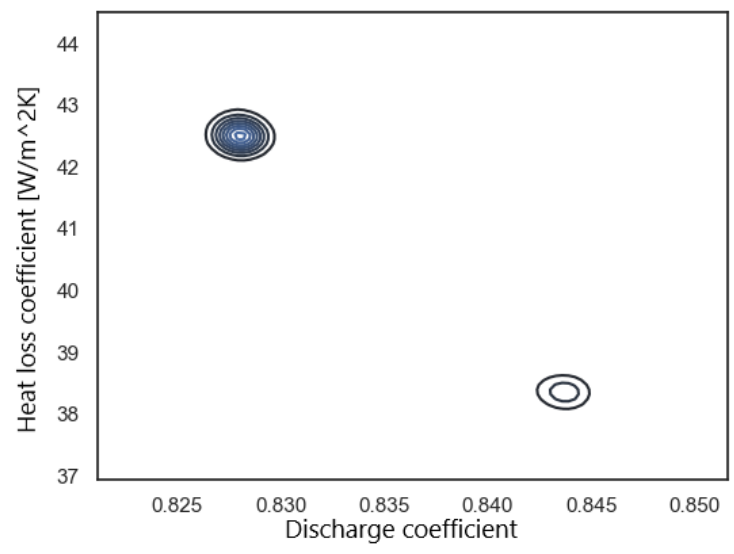

Figure 6. Bivariate posterior distributions for experiment 11.

Due to uncertainty in measurements, in general, it is not possible to uniquely determine the values of the unknown parameters. In the Bayesian approach, this problem is addressed by the posterior distribution. The posterior distribution summarizes the belief in the probable range of the values for the unknown parameters (Kruschke, 2015). Models are simplified versions of reality. In this context, the simplification means that we only take into account certain aspects of the reality and assume that the other aspects either cancel out each other or have no significant influence on the description or predicted behaviour of the system of interest. One way to evaluate how good these simplifications are is to check how accurately the model mimics the data. Therefore, it is important to not confuse the model inadequacies with the uncertainty due to inference. Thus, it is important to keep track of the sources of uncertainty. One way to do this is by conducting the so called posterior predictive check.

The MCMC results have been applied into the PPP model (Figure 7). Both experimental and simulation results have their uncertainty, included in curves. For experimental results, the uncertainty is $\pm 1 \%$ FSO BFSL (grey area around black line Figure 1). For the simulation, the uncertainty was calculated at the specific time for each sample (vertical red lines from blue dots Figure 7). The simulation overestimates the actual pressure with $0.6 \mathrm{kPa}$ in maximum pressure and underestimates in minimum pressure is $0.6 \mathrm{kPa}$ as well which is acceptable.

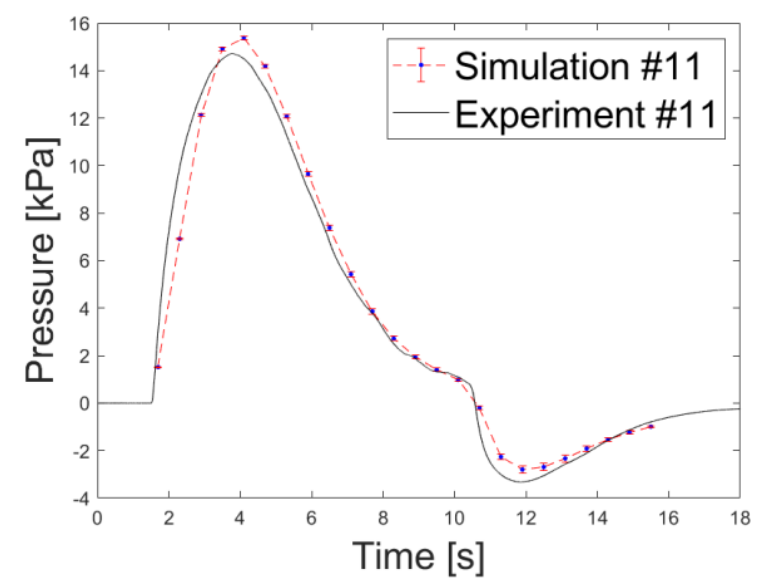

Figure 7. Pressure dynamics in $14,9 \mathrm{~m}^{3}$ the enclosure during experiment 11: experimental (black line), simulation with estimated $\boldsymbol{\theta}$ individualy for each time step (blue dots). The standard deviation is represented by error bars (red lines with caps).

The simulation result shows good accuracy with experiments (Figure 8). 


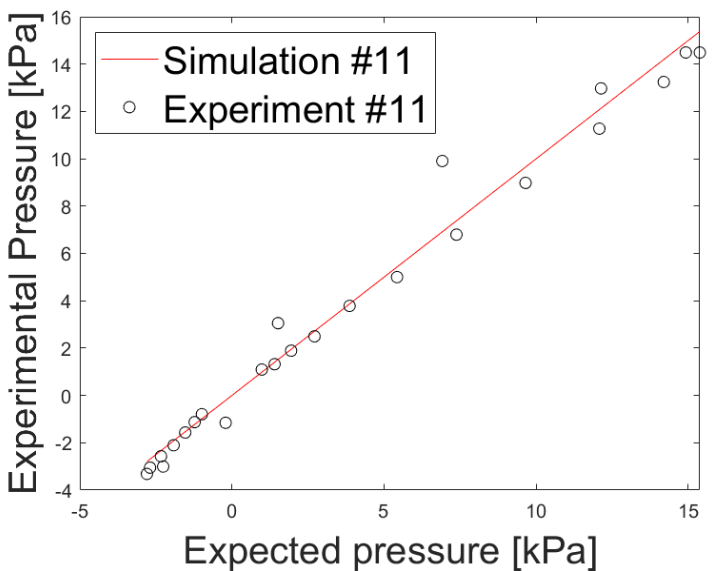

Figure 8. Exp11 pressure predicted (red line) vs. measured (black circles).

\section{Summary}

In this paper, we have used a Bayesian approach to estimate parameters in a PPP model. The intended use for this model is to simulate overpressures from accidental hydrogen releases in confined spaces. The parameters of interest were the heat loss coefficient and the discharge coefficient. The data used in creating the model, and used when estimating the parameters, was collected from large scale experiments performed by the University of South-Eastern Norway.

Markov Chain Monte Carlo was used to generate samples from the posterior distribution. The performance of the MCMC algorithm was evaluated, and seen to perform well.

In this analysis, we found that a discharge coefficient of $C=0.9$ and a heat loss coefficient of $h_{\text {loss }}=30$ are the most likely values which capture the results across all the experiments. The Bayesian analysis of the model gave the most probable values for performed experiments (set up dimension and flow rates).

\section{Acknowledgements}

The authors wish to acknowledge the European Union Fuel Cells and Hydrogen 2 Joint Undertaking (JU) under grant agreement No 826193 with support from European Union's Horizon 2020 for funding and supporting the HyTunnel project.

This work was performed within MoZEES, a Norwegian Centre for Environment-friendly Energy Research (FME), co-sponsored by the Research Council of Norway (project number 257653) and 40 partners from research, industry and public sector.

We thank Andre V. Gaathuag for his work on the PPP model and Ali Ghaderi for comments and supervision that greatly improved the manuscript

\section{References}

S. Brennan, D. Makarovand, and V.V. Molkov. Dynamics of Flammable Hydrogen-Airixture Formation in an
Enclosure with a Single Vent. In Proceedings -Fire and Explosion Hazards 6, 11.-16. April, Leeds, UK, 493-503, 2010. doi: 10.3850/978-981-08-7724-8_07-04.

S. Brennan and V.V. Molkov. Pressure peaking phenomenon for indoor hydrogen releases. International Journal of Hydrogen Energy, 43(39), 18530-18541,2018. doi: 10.1016/J.IJHYDENE.2018.08.096

D.A. Crowl and J.F. Louvar. Chemical Process Safety Fundamentals with Applications, 3rd ed.; In B. Goodwin, J. Fuller and E. Ryan, editor. Prentice Hall 2011.

F. Daly, D. Hand, M. Jones, A. Lunn, and K. McConway. Elements of Statistics (1st ed.). Addison Wesley, The Open University, 1995.

H.G. Hussein, S. Brennan, V. Shentsov, D. Makarov, and V.V Molkov. Numerical validation of pressure peaking from an ignited hydrogen release in a laboratory-scale enclosure and application to a garage scenario. International Journal of Hydrogen Energy, 43(37), 17954-17968, 2018. doi: 10.1016/J.IJHYDENE.2018.07.154.

J.K. Kruschke. Doing Bayesian Data Analysis, A tutorial with R, JAGS and Stan. In Becoming a Brilliant Trainer (2nd ed.) 2015. doi: 10.4324/9781315627960-16.

A.W. Lach. Pressure Peaking Phenomena-unignited releases. Internal Report experiments, 2019.

D. Makarov,V. Shentsov, M. Kuznetsov, and V.V Molkov. Pressure peaking phenomenon: Model validation against unignited release and jet fire experiments. International Journal of Hydrogen Energy, 43(19), 9454-9469,2018. doi: 10.1016/J.IJHYDENE.2018.03.162.

MathWorks. Matlab User Guide(r2020b). ode45 Apple Hill Drive Natick, MA, 2020.

B.J. Mcbride, S. Gordon, and M. Reno. Coefficients for Calculating Thermodynamic and Transport Properties of Individual Species. Nasa Technical Memorandum, 4513(NASA-TM-4513), 98, NASA, US. Government 1993.

J. A. Vrugt. Markov chain Monte Carlo simulation using the DREAM software package: Theory, concepts, and MATLAB implementation. Environmental Modelling \& Software, 75, 273-316, $2016 . \quad$ doi: 10.1016/J.ENVSOFT.2015.08.013.

J. Wang, Z. Zhou, K. Lin, C.K. Law, and B. Yang. Facilitating Bayesian analysis of combustion kinetic models with artificial neural network. Combustion and Flame, 213, 8797,2020. doi: 10.1016/j.combustflame.2019.11.035. 